\title{
Percepciones sobre los medios de comunicación de profesores universitarios de la educación infantil en Chile
}

\section{Perceptions about media in teacher trainers of early childhood education in Chile}

\author{
Pablo Andrada*, Cristian Cabalin**
}

*Universidad Central de Chile

**Universidad de Chile \& Universidad Central de Chile

Resumen

\begin{abstract}
Cada vez más los avances tecnológicos como los televisores inteligentes, computadores, tablets o pizarras digitales interactivas son parte del inventario de los jardines infantiles. Estas adquisiciones no han ido acompañadas de una educación mediática que permita que los niños y niñas aprendan a convivir con los medios desde la educación infantil. El presente estudio tiene como objetivo conocer cuáles son las percepciones sobre los medios de comunicación que tienen los profesores universitarios de la educación infantil chilena, lo que permitirá, a su vez, aproximarnos a establecer cómo es incorporada la educación mediática en la formación docente de este nivel educativo. Para ello, se aplicó un cuestionario a distancia a profesores de universidades chilenas que imparten cursos relacionados con los medios de comunicación en carreras de educación infantil. Los resultados muestran que los profesores que enseñan sobre lenguaje y comunicación responden más a la tradición apocalíptica de los medios, mientras los de TIC siguen la
\end{abstract} tradición apologética.

Palabras clave: educación mediática, formación docente, educación infantil, TIC, percepción, educación universitaria.

Abstract

Increasingly, technological advances have made smart televisions, computers, tablets or interactive whiteboards are part of the inventory of kindergartens. Despite some attempts to keep the kindergartens free from the presence of the media, the desire to take advantage of technological advances has made televisions, computers, tablets and electronic blackboards being part of the inventory of these schools. These acquisitions have not been accompanied by a media education that allows children to learn to live with the media from early childhood education. The present study has as objective to know what the perceptions about the means of communication are that professors of Chilean children's education have, which will allow us, in turn, to approximate to establish how media education is incorporated in teacher training of this educational level. To this end, a distance questionnaire was applied to professors from Chilean universities who taught courses in media relations in early childhood careers. The results show that professors who teach language and communication respond more to the apocalyptic tradition of the media, while those of ICT follow the apologist tradition.

Keywords: media education, teacher training, early childhood education, ICT, perception, university education.

Submitted: $6^{\text {th }}$ June 2018

Accepted: $8^{\text {th }}$ March 2019

\section{How to quote this article:}

Andrada, P., Cabalin, C. (2019). Percepciones sobre los medios de comunicación de profesores universitarios de la educación infantil en Chile. Observatorio, 13(2), 106-124. 


\section{Introducción}

En la primera función de cine de la historia, los hermanos Lumière presentaron siete registros de situaciones que se vivían en 1895. El sexto registro mostraba un tren expulsando humo y acercándose a la pantalla. El impacto del público fue tal que muchas personas salieron corriendo de la sala de exhibición asustadas, pensando que el tren las atropellaría; otras se quedaron disfrutando de ese espectáculo y presenciaron el último registro donde vieron dos veces el derribo de una pared por parte de unos obreros. La segunda vez se mostró la escena en retroceso por lo que los espectadores que permanecieron en la sala fueron testigos de los primeros efectos especiales del cine. En las reacciones de esta primera función apreciamos las dos formas en que posteriormente nos relacionaríamos mayoritariamente con los medios: el pánico y la fascinación.

La prehistoria de la educación mediática la encontramos en el análisis estético de los cineclubes en la década de 1920. Estaba presente en el deseo reflexionar sobre lo que mostraba la pantalla grande y de "educar" acerca del cine. Ya "en 1922, se celebró en Francia la primera conferencia nacional de los departamentos regionales de educación cinematográfica" (Fedorov, 2011, p. 8). Una vez que la pantalla se apagaba, muchas personas sentían la necesidad de conversar sobre lo que habían visto. Los más entusiastas se organizaron para compartir funciones y hablar sobre las propuestas artísticas de un director, la estética de un filme o la situación social que se plasmaba en el celuloide.

El análisis estético del cine llegó a las aulas a partir de la década de 1950 en Francia, Canadá, Inglaterra y Estados Unidos con cursos que buscaban educar a los estudiantes en el lenguaje audiovisual. No se hablaba de educación mediática, pero se enseñaba a apreciar el medio de comunicación más consumido de la época. Se realizaban clases desde la fascinación que producía el séptimo arte, reconociendo los méritos de un autor y de su obra, siendo parte de la tradición que se siente atraída por los medios o apologista. No obstante, el análisis estético fue criticado por establecer una línea divisoria entre una alta y una baja cultura, despreciando producciones populares catalogadas como cine de mala calidad por no cumplir con los gustos estéticos canonizados (Fedorov, 2011), por lo que también podemos ubicar el análisis estético del cine en la tradición del pánico y rechazo a los medios o apocalíptica.

A partir de la década de los 70, el cine pierde hegemonía como espectáculo de masas y el interés académico se amplía hacia los nuevos medios, en especial, la televisión y la radio. A continuación, daremos cuenta de las tradiciones epistemológicas que surgen de la relación entre la educación y los medios de comunicación, lo que nos permitirá sentar las bases para abordar el objetivo de realizar una aproximación a la forma cómo es incorporada la educación mediática en la formación docente de la educación infantil en Chile.

\section{Tradición apocalíptica}

Los medios de comunicación y las tecnologías pueden ser percibidos como amenazas, ya sea porque generan daños en la salud, manipulan lo que piensan las personas o simplemente porque consumirlos, interaccionar y expresarse mediante ellos es visto como una pérdida de tiempo. En el extremo de esta visión, encontramos sentimientos como el pánico o la amenaza, lo que se ha conocido como la tradición apocalíptica de los medios. 
El modelo inoculativo o moralizante es la versión más extrema de la tradición apocalíptica de los medios. Este modelo se basa en la idea de que los productos mediáticos pueden incluir imágenes o mensajes que tienen una influencia negativa en la población, por lo que es necesario proteger a los espectadores de contenidos inmorales tales como la violencia y el sexo. Desde esta perspectiva, los medios tendrían un gran poder de persuasión sobre las personas y se hace necesario restringir su exhibición y recepción. Ya en la década de 1910 las autoridades comenzaban a censurar películas, determinando qué filmes debían ser proyectados. Se crean agrupaciones civiles que fiscalizan el contenido audiovisual. En 1936, el Papa Pío XI realiza una encíclica sobre espectáculos cinematográficos donde felicita el trabajo de la denominada Liga de la Decencia que contribuyó "al establecimiento de un código de censura bastante estricto en 1934" (Purcell, 2011, p. 195).

En 1938 Orson Welles narró en la radio una adaptación de la novela de ciencia ficción La Guerra de los Mundos, donde un grupo de alienígenas invade la Tierra. Miles de auditores entraron en pánico creyendo que la historia era real porque sintonizaron el programa luego de la introducción donde se explicaba que lo relatado era una ficción. El miedo colectivo generó un colapso de las líneas telefónicas y mostró el poder de influencia de los medios sobre las personas, dando fuerza a la idea de que la población debe ser protegida de ellos.

En 2008, el Consejo Superior del Audiovisual en Francia prohibió a los canales de televisión abierta emitir programas para niños menores de tres años. La medida se fundamentó entre otras cosas en que "una sobreexposición a la televisión podría provocar una hiperestimulación con dificultades para dormir, hiperactividad y falta de concentración; temor incluso a una sobreestimulación de incitaciones tecnológicas que (se teme) podría generar desarrollos cerebrales desbalanceados" (Fuenzalida, 2016, p. 43).

Los principales voceros de esta tradición son los reivindicadores, grupo compuesto por activistas, políticos, expertos y comentaristas, que tienden a responsabilizar a los medios de problemas sociales más complejos. Ellos logran la atención de los medios a través de la exageración de los problemas y el uso de evidencia científicas de carácter dudoso (Buckingham, 2013). Algunos de sus superventas critican la influencia del marketing dirigido a los niños, tales como Born to Buy. The commercialized Child and the New Consumer Culture (2004) o Consuming kids: The Hostile Takeover of Childhood (2004).

"Además de convertir a los niños en consumidores prematuros, se acusa a los media de promover el sexo y la violencia, la comida basura, las drogas, el tabaco y el alcohol, los estereotipos de género y falsos valores morales, así como de contribuir a una "epidemia" de trastornos de salud mental, ansiedad, estrés y adicciones dañinas (incluyendo la misma adicción al consumo)" (Buckingham, 2013, p. 20)

En una columna de opinión de 2016 en el periódico The Sun, Sue Palmer, la autora del superventas Toxic Childhood, hace un llamado a las autoridades británicas a establecer el máximo de horas que los niños deben interactuar con las pantallas. "Necesitamos urgentemente pautas de salud pública sobre cuánto debemos permitir que nuestros niños usen dispositivos digitales. Si bien la tecnología basada en pantalla es excelente para los adultos, no es buena para los niños pequeños" (Palmer, 2016).

El modelo inoculativo ha instalado la idea de que los medios de comunicación son una amenaza para la educación de los niños y jóvenes, llevándolos a perder el tiempo mirando contenidos inmorales o superficiales. En la actualidad el modelo no se expresa mediante documentos como encíclicas papales 0 políticas explícitas sobre mantener los medios de comunicación fuera de las aulas. Se encuentra en el sustrato social y se alimenta de los reivindicadores apocalípticos de los medios que, paradójicamente, utilizan 
a éstos para propagar su discurso sobre los peligros que conlleva el uso de las pantallas para las personas, en especial para los niños.

Otra vertiente de la tradición apocalíptica de los medios la encontramos en el modelo crítico, el cual se caracteriza por "leer" de forma crítica los medios de comunicación, buscando develar las ideologías y valores presentes en sus mensajes. Su precursor es el educador brasilero Paulo Freire, quien propuso la superación de la educación tradicional que forma sujetos pasivos que reproducen la cultura dominante. Este modelo educativo busca liberar a los estudiantes, alfabetizando a ciudadanos con pensamiento propio que primero aprender a "leer" el mundo y luego la palabra (Freire, 2005).

Esta perspectiva educativa tuvo gran repercusión en Latinoamérica en la década de los 60 en un contexto donde las sociedades de esta región buscaron liberarse del dominio económico y cultural de los países desarrollados. En el ámbito de los medios, el afán estuvo centrado en revelar los mecanismos de control ideológico de la industria cultural estadounidense. El libro Para Leer al Pato Donald (Dortman y Mattelard, 2015) es uno de los íconos de la mirada crítica de esa época mostrando como se impone la ideología capitalista en un producto mediático aparentemente inocente.

"La motivación de este mundo excluye el amor. Los niñitos admiran a un lejano tío, que descubrió 'un invento que mata al gusano de la manzana'. Aseguran: 'el mundo entero le está agradecido por ello... Es famoso... y rico'. Donald responde acertadamente: 'iBah! El talento, la fama y la fortuna no lo son todo en la vida' -'¿No? ¿Qué otra cosa queda?', preguntan Hugo, Paco y Luis al unísono. Y Donald no encuentra nada que decir, sino: 'Er... Humm... A ver... Oh-h'"' (p. 29)

Durante la década de los 80, Mario Kaplún fue uno de los mayores promotores de la lectura crítica de los medios. Siguiendo las ideas de Freire, propone un modelo comunicativo liberador, donde "un óptimo recurso para generar esa actitud crítica respecto de los medios de comunicación consiste en hacer que los propios educandos los practiquen y descubran así por ellos mismos las operaciones manipulatorias habilitadas por las mediaciones comunicacionales" (Kaplún, 2002, p. 217). A partir de la propuesta de Jean Cloutieu de una comunicación participativa realizadas por sujetos que son emisores y receptores (Emirec), Kaplún formula un modelo donde el destinario esté al comienzo del proceso, permitiendo una comunicación popular donde los mensajes representen y reflejen a los destinatarios. "Proponemos Ilamar prealimentación a esa búsqueda inicial que hacemos entre los destinatarios de nuestros medios de comunicación para que nuestros mensajes los representen y reflejen. Por ahí comienza y debe comenzar un proceso de comunicación educativa" (Kaplún, 2002, pp. 73-74).

La lectura crítica de los medios es uno de los pocos enfoques que encontramos para estudiar los medios en la escuela. Las escasas presencias de educación mediática en la educación formal que encontramos en la actualidad se centran en analizar la ideología y valores. Un ejemplo son los estándares orientadores de educación parvularia (infantil) de Chile, donde se espera que "la educadora de párvulos que ha finalizado su formación inicial demuestra el logro de este estándar cuando: (...) 7. Lee en forma crítica los mensajes de los medios de comunicación de masas" (Mineduc, 2012, p. 33).

Otro caso lo encontramos en España, donde se analizaron referencias bibliográficas de asignaturas relacionadas con la educación mediática en facultades de educación y comunicación. Los resultados mostraron que los aspectos que más se valoraban era la comprensión de la función social de las tecnologías y "la capacidad de detectar la ideología y los valores, explícitos o latentes, incluso en comunicaciones 
inadvertidas, adoptando una actitud crítica ante ellos". Esta última capacidad se vincula directamente con la tradición de la lectura crítica de los medios (Aguaded-Gómez, López \& Cruz-Díaz, 2015, p. 55)

Esta lectura conlleva una desconfianza general a los productos mediáticos, porque se los percibe como mecanismos de poder que legitiman y promueven la reproducción de la cultura dominante. Por ello, se sitúan en una posición apocalíptica donde más que el temor predomina el rechazo a los medios.

\section{Tradición apologética}

La aparición de una nueva tecnología genera mucho entusiasmo. En las visiones más extremas se las señala como revolucionarias y certificadoras de la defunción de la tecnología anterior. El cine acabaría con los libros, la televisión con el cine e Internet lo hará con la televisión. Sin embargo, la realidad nos muestra que no ha existido una sustitución sino procesos de convergencia entre los medios y las tecnologías (Jenkins, 2008). Los medios de comunicación pueden ser vistos como tecnologías o recursos que complementan el trabajo docente haciéndolo más eficiente o ayudando a motivar a los estudiantes. La televisión educativa se enmarca en esta perspectiva. Sus primeras manifestaciones fueron las teleclases, donde se encendía el televisor en el aula para que los estudiantes aprendieran a distancia sobre temas específicos. "Se pretendía hacer coincidir los horarios de clases escolares, especialmente en las mañanas, con programas de televisión estrictamente relacionados con determinadas materias escolares, para emitirlos en las aulas escolares" (Fuenzalida, 2005, p. 14).

El reemplazo del docente por el aparato televisivo se sustentaba en el aumento de la cobertura del sistema escolar mediante la educación a distancia y el atractivo que tendría el lenguaje audiovisual para los estudiantes. Al poco tiempo, en especial en Latinoamérica, se abandonaron esta forma de clases y los contenidos quedaron almacenados en videocasetes que podrían servir de apoyo para los docentes. Esta modalidad tampoco tuvo éxito debido a: la carencia de material audiovisual curricularizado, la falta de acceso a videocasetes y la escasa formación docente para trabajar con material audiovisual (Fuenzalida, 2005). También existen casos de televisión educativa para adultos. Desde la década del 80 hasta 2003, la Pontificia de la Universidad Católica de Chile produjo y transmitió el programa de televisión educativa Teleduc, donde se enseñaba a adultos sobre comunicación efectiva, instalaciones eléctricas, negocios, fotografía, entre otros. Sin embargo, con la masificación de la informática se acabó este programa, Teleduc dio un giro tecnológico, concentrando su formación a distancia a través del lenguaje digital mediante cursos realizados por plataformas online.

El desarrollo de la informática ha impulsado una nueva apología de los medios. Muchos gobiernos hayan querido dar el "salto tecnológico" creando aulas de informática en las escuelas, dejando de lado la enseñanza del audiovisual. Con esta inversión se ha buscado disminuir la brecha digital, permitiendo que la población de menos recursos tenga acceso la informática y a Internet. Dicho de otra forma, se ha buscado alfabetizar digitalmente a la población en el entendido que en la llamada sociedad de la información es imprescindible manejar correctamente las Tecnologías de la comunicación y la Información (TIC). Un caso emblemático ha sido el plan Ceibal, en Uruguay con la entrega de un computador a cada estudiante en edad escolar (en inglés one laptop per child). Este correcto manejo de la tecnología se suele llamar competencia digital o habilidades TIC para el aprendizaje y se refiere a "la capacidad para resolver problemas, crear innovaciones, 
gestionar y hacer uso crítico de información y colaborar con otros en el ambiente digital, así como participar de manera responsable en las diferentes esferas de la vida social que hoy se despliegan de manera predominante a través de medios y dispositivos digitales" (Mineduc, 2017, p. 4).

La publicación de EI Marco de competencias de los docentes en materia de TIC por parte de la Unesco, en 2008, fue un hito que validó internacionalmente la inclusión de las tecnologías en la formación docente. Este marco incluye tres enfoques didácticos escalonados: el aprendizaje de los elementos básicos de la tecnología, la profundización del conocimiento y la creación de conocimiento. Este marco tuvo eco en Chile. En 2011 se publica el documento Competencias y Estándares TIC para la Profesión Docente, el cual forma parte del Marco de Competencias TIC para el sistema escolar que incluye documentos sobre competencias TIC para directores de escuela, jefes de Unidades Técnico Pedagógica (UTP), orientadores y coordinadores de bibliotecas.

También las TIC están presentes en el currículo. Los estudiantes chilenos desde séptimo básico a segundo medio (12 a 15 años) cursan la asignatura de tecnología. Al concluir el curso, se espera que sean capaces de "Usar y evaluar tecnologías de la información y la comunicación de forma responsable y respetar los límites legales, éticos y culturales de compartir información, reconociendo las oportunidades, riesgos e impactos que puede implicar su uso" (Mineduc, 2015, p. 379).

A pesar de estas intenciones en alfabetización tecnológica, las primeras evaluaciones sobre competencia digital en Chile muestran que más de la mitad de los estudiantes no alcanzan los mínimos en la gestión de la información y la utilización de las herramientas digitales para compartir y colaborar (Mineduc, 2014). Debido a estos resultados, se creó un Consejo Asesor para la Agenda Digital en Educación que ha señalado que para mejorar los resultados los esfuerzos futuros no deben estar en el uso de la tecnología sino en experiencias de aprendizajes significativas (Mineduc, 2017).

En el ámbito académico existiría un consenso respecto de que la promoción de las TIC se ha realizado desde un enfoque reduccionista, donde la competencia digital se centra en aspectos técnicos vinculados al uso y manejo de la tecnología. "Para una alfabetización puramente instrumental y tecnológica no es imprescindible la escuela. Es más, actualmente estas destrezas básicas de manejo de tecnología se adquieren en su mayor parte en entornos no escolares" (Gutiérrez y Tyner, 2012, p. 32). La nueva versión de las competencias TIC de la Unesco pareciera haber tomado nota de estas críticas, puesto que ya no se habla tanto de la búsqueda e información, sino que se apunta a la apropiación de la tecnología a través de la integración, reorientación y evaluación de ella por parte de los docentes, buscando un uso reflexivo de las TIC (ValenciaMolina, et al., 2016).

\section{Convivir con los Medios: una tercera vía}

En Chile, un $82 \%$ de la población posee un teléfono celular que se puede conectar a Internet y un $74 \%$ posee servicio de televisión por cable o satelital. Si nos enfocamos en los niños menores de siete años, un $35 \%$ de ellos tienen objetos tecnológicos propios, destacando el Tablet con un $22 \%$. Además, según el $76 \%$ de los padres chilenos, es muy fácil que los niños menores de 13 años accedan mediante Internet a contenidos inadecuados, como videos que promueven el racismo o la violencia (CNTV, 2017). Hacerse cargo de esta realidad es una tercera vía para relacionarse con los medios. Así como en el pasado uno de los grandes 
desafíos educativos fue el que la mayoría de la población aprendiera a leer y escribir, uno de los retos actuales es que las personas aprendan a convivir de manera positiva con los medios. Esta tercera vía se conoce como educación mediática y se puede definir como la que permite "interaccionar de manera crítica con mensajes (mediáticos) producidos por los demás y siendo capaz de producir y de diseminar mensajes propios" (Ferrés \& Piscitelli, 2012, pp. 77-78). Se sustenta en las cifras que muestran que los medios de comunicación forman parte de la vida de las personas, tal como lo señalaba ya hace 35 años la Declaración de Grünwald sobre la Educación Relativa a los Medios de Comunicación.

"Vivimos en un mundo en que los medios de comunicación son omnipresentes: un número de individuos cada vez mayor consagra buena parte de su tiempo a mirar la televisión, a leer diarios y revistas, a escuchar grabaciones sonoras o la radio. En ciertos países, por ejemplo, los niños pasan ya más tiempo ante la pantalla de televisión que en la escuela" (Unesco, 1982, p. 1).

La educación mediática se manifiesta a través de distintos ámbitos. En el legislativo, el Parlamento Europeo aprobó en 2008 una resolución sobre la alfabetización de los medios de comunicación en un mundo digital donde señaló que la gran concentración de los medios de comunicación "puede poner en peligro el pluralismo de los mismos y que, por ello, la alfabetización mediática es de capital importancia para la formación política y la participación activa de los ciudadanos de la Unión" (Resolución 2008/2129).

En la resolución se señala que la educación mediática debe incluirse en la formación de profesores de todos los niveles de la educación escolar; en las escuelas, estando presente en los planes de estudios; en las universidades, promoviendo la educación mediática en la formación profesional y en la formación continua, y también en las personas mayores, buscando eliminar los temores que generan los medios y las nuevas tecnologías en este grupo (Resolución 2008/2129).

En el ámbito de la docencia, un esfuerzo relevante ha sido el documento Alfabetización Mediática $e$ Informacional. Currículo para profesores, de la Unesco. En el texto se busca unir la tradición epistemológica que busca entender las funciones de los medios de comunicación, tener una visión crítica sobre ellos y poder usarlos para expresarse, conocida como alfabetización mediática, con la tradición que se preocupa del acceso, evaluación y uso ético de la información, conceptualizada como alfabetización informacional. El currículo permite que los profesores desarrollen un programa de estudios sobre el conocimiento y entendimiento de los medios e información para los discursos democráticos y la participación social, la evaluación de los textos mediáticos y fuentes de información, y la producción y uso de los medios y la información (Wilson, Grizzle, Tuazon, Akyempong \& Cheung, 2011).

A nivel de los estados, destaca la propuesta curricular de Portugal con su Referencial de Educação para os Media para a Educação Pré-escolar, o Ensino Básico e o Ensino Secundário (2014), realizado por investigadores del Centro de Estudos de Comunicação e Sociedade, de la Universidade do Minho, para el Ministerio de Educación Portugués. El referencial fue construido tanto a partir de documentos de educación mediática internacionales como desarrollados previamente en Portugal. A partir de temas, subtemas y descriptores de desempeños, los profesores pueden desarrollar un conjunto de conocimientos, capacidades, actitudes y comportamientos en estudiantes de educación infantil, primaria y secundaria, que van desde 
comprender el mundo actual, las TIC y las pantallas, la publicidad y las marcas hasta entender las audiencias, los públicos y el consumo (Pereira, Pinto, Madureira, Pombo \& Guedes, 2014).

Si tomamos como hito fundador la declaración de Grünwald, la educación mediática cumple 35 años. Gracias al aporte de destacados educadores e investigadores (entre ellos, Freire, Cloutier, Vallet, McBride, Kaplún, Masterman, Livingstone, Buckingham, Ferrés, Pinto y Fuenzalida, entre otros) han existido avances en el ámbito del reconocimiento internacional de este campo de estudio, impulsado principalmente por la Unesco, y en la construcción de currículos que apoyen la implementación de la educación mediática en la escuela. Sin embargo, sigue pendiente su implementación continua desde el nivel infantil hasta el universitario.

\section{Docentes y Medios}

En 2007, la Declaración de Paris o 12 recomendaciones para la educación mediática, de la Unesco (2007), enfatizó la importancia de la formación inicial de los profesores (recomendación 4) y el desarrollo de una didáctica apropiada (recomendación 5). Esta declaración, realizada 25 años después de la de Grünwald, señala que los docentes son un factor clave para la educación mediática. "Initial training of teachers is a key element of the system and must include theoretical dimensions and practical skills; it needs to be based on a good knowledge of young people's media uses" (Unesco, 2007, p. 2).

Las recomendaciones no han logrado concretarse en planes sistemáticos que incidan en la formación de los docentes y los estudiantes que permitan contrarrestar y complejizar las tradiciones apocalípticas y apologéticas de los medios de comunicación.

Los estudios muestran que hay una baja oferta de educación mediática en la formación de los docentes y cuando existe se entrega desde una perspectiva que se reduce a la dimensión del uso de la tecnología. En el caso de España, solo un $21 \%$ de los grados del ámbito de la educación que se ofrecen en las universidades tienen una asignatura relacionada directamente con la enseñanza de los medios (Ferrés, Masanet y Blanco, 2014). Al analizar los manuales universitarios más utilizados en las facultades de educación y comunicación, se comprobó que en las escasas ocasiones en que existe educación mediática en la formación docente de los profesores ésta se concentra en la dimensión del uso de la tecnología y de la ideología y valores, menoscabando principalmente la dimensión estética (López \& Aguaded, 2015).

Las tecnologías y los medios parecieran ir por distintos caminos en la formación docente. Las TIC son incluidas en los planes de formación docente en las universidades y en la escuela como una asignatura específica, entregando prestigio y un aura de modernidad a los centros educativos, mientras "el estudio de los medios y la educación para los medios ha venido luchando sin demasiado éxito por hacerse un hueco en los currícula de la enseñanza obligatoria en distintos países" (Gutiérrez y Tyner, 2012). En la mayoría de los países hay una ausencia de un plan continuo de educación mediática que vaya desde la educación infantil hasta la secundaria, tal como pedía la declaración de Grünwald (Unesco, 1982). Por el contrario, es abordada de forma transversal, convirtiéndose la mayoría de las veces en "tierra de nadie".

En Chile, donde la educación mediática es solo un contenido del currículo de la asignatura de Lenguaje y Comunicación de la enseñanza secundaria, los profesores son autodidactas en aprender acerca de la educación mediática y también cómo enseñarla. Utilizan los medios que les resultan más cercanos como la 
prensa, relegando el lenguaje audiovisual. Esta falta de formación redunda en que, a pesar de que el currículo busca profundizar en el estudio de los medios, la mayoría de las veces son utilizados como recurso metodológico, es decir, para aprender otras cosas (De Fontcuberta, 2009). Subyace en ellos la errónea idea de que basta consumir medios para poder enseñar sobre ellos.

Una vez revisadas las tradiciones epistemológicas de la relación entre la educación y los medios de comunicación, podemos abordar el objetivo de conocer cuáles son las percepciones sobre los medios de comunicación que tienen los profesores universitarios de la educación infantil chilena.

\section{Metodología}

Para cumplir el objetivo de realizar una aproximación a la forma cómo es incorporada la educación mediática en la formación docente de la educación infantil en Chile, se realizó una metodología mixta. Se aplicó un cuestionario a distancia a 30 profesores universitarios de carreras (grados) de educación parvularia (infantil) de universidades chilenas que impartían asignaturas donde se estudian o utilizan medios de comunicación. Las preguntas fueron cerradas (parte cuantitativa) y abiertas (parte cualitativa). El universo de esta investigación fueron las 46 licenciaturas de educación parvularia que se ofrecieron en Chile en 2016. Se invitó a participar a todas las universidades, obteniendo respuesta positiva de 16 universidades. En el caso de los profesores, 11 universidades participaron efectivamente, lo que constituye la muestra final y representa el $23 \%$ del universo de universidad que ofrecen las carreras.

La muestra no es representativa, pero sí nos permite dar cuenta de una tendencia de la formación docente en educación en medios en la educación infantil en Chile. De las 11 universidades que participaron en el estudio, ocho son de la capital del país, Santiago, y tres de regiones, específicamente de las ciudades de La Serena, Valparaíso y Talca. De esta forma contamos con 21 profesores de la capital y 9 de regiones.

Tabla 1: Composición de la muestra del estudio

\begin{tabular}{|l|l|l|}
\hline Universidad & Región & Número de profesores \\
\hline Alberto Hurtado & Metropolitana & 4 \\
\hline Andrés Bello & Metropolitana & 3 \\
\hline Finis Terrae & Metropolitana & 6 \\
\hline Pedro de Valdivia & Metropolitana & 1 \\
\hline Pontificia UC & Metropolitana & 1 \\
\hline U. de Chile & Metropolitana & 2 \\
\hline U. de las Américas & Metropolitana & 1 \\
\hline U. del Desarrollo & Metropolitana & 3 \\
\hline La Serena & La Serena & 5 \\
\hline Pontificia UC Valparaíso & Valparaíso & 2 \\
\hline UC Maule & Maule & 2 \\
\hline Total & & 30 \\
\hline
\end{tabular}

Fuente: Elaboración propia 
En el cuestionario se preguntó a los profesores sobre la percepción que tenían sobre los medios de comunicación y el uso que hacían de ellos. En este texto solo daremos cuenta de lo referido a la percepción. Referido a ésta, se incluyeron cuatro ítem de preguntas, una de ellas abierta la que corresponde a la parte cualitativa del instrumento. Los temas abordados fueron las percepciones respecto del rol de los medios en la sociedad, donde debían señalar su acuerdo con las ideas de que los medios son el principal vehículo de socialización en la actualidad, que pueden ayudar a la autonomía personal y la transformación social y que informan de manera objetiva sobre los hechos que suceden.

Otro ítem fue el referido a la influencia de los medios en las personas, en el que expresaron su acuerdo con que los medios ejercen menos influencia cuando su función es solo entretener o ayudar a "pasar el rato", influyen más cuando apelan solo a lo emocional e inconsciente de las personas y ejercen una influencia mayor cuando entregan elementos cognitivos y racionales

Un tercer tema abordado fue la forma en que se debieran incorporar la enseñanza de los medios en la formación de los y las profesionales de la educación infantil, preguntando si debía ser mediante una asignatura específica, un contenido de una asignatura, un contenido transversal, de otra forma o si no debiera ser incorporada

Finalmente, se preguntó a los profesores si consideraban relevante que se enseñara sobre medios de comunicación a los niños en los jardines infantiles, siendo este último aspecto de respuesta abierta.

El análisis de las respuestas de los 30 profesores fue descriptivo y cualitativo. La descripción se realizó estableciendo los porcentajes de acuerdo y desacuerdo con las ideas y consultas realizas. La pregunta abierta fue examinada con la herramienta Nvivo 11 Pro, lo que permitió organizar en categorías los motivos por los que se justificaba la inclusión o no de los medios de comunicación en los jardines infantiles.

También se realizó un segundo análisis bajo la hipótesis de que el tipo de asignatura que se enseñaba influía en la percepción que se tenía de los medios de comunicación. El segundo análisis puso el foco en 11 profesores: seis de asignaturas TIC y cinco, de Lenguaje y Comunicación y 19 de Otras Asignaturas. Los resultados permitieron establecer perfiles de las percepciones de los profesores sobre los medios según el tipo de asignatura que enseñan.

Cabe que el grupo de profesores de Otras Asignaturas es amplio y no se buscaron establecer conclusiones sobre ellos. El foco del análisis estuvo en los profesores TIC, como representantes de la alfabetización digital, y los de Lenguaje y Comunicación como los que pueden enseñar de medios de comunicación en los referido, precisamente, a la comunicación mediada.

Tabla 2: Tipos de profesores según la asignatura que enseñan

\begin{tabular}{|l|l|}
\hline $\begin{array}{l}\text { Tipo de } \\
\text { Asignaturas }\end{array}$ & Nombre Asignaturas impartidas por profesores* \\
\hline TIC & $\begin{array}{l}\text { Informática educativa, Habilidades Básicas TIC, Tecnología Integrada a la Educación } \\
\text { Parvularia, Tecnología de la Información en el Contexto Educativo, Multimedia educa- } \\
\text { tiva. }\end{array}$ \\
\hline $\begin{array}{l}\text { Lenguaje y } \\
\text { Comunicación }\end{array}$ & $\begin{array}{l}\text { Adquisición y desarrollo de la lengua, Módulos de mención lenguaje, Habilidades del } \\
\text { Pensamiento, Fundamentos y desarrollo del lenguaje, Desarrollo del lenguaje }\end{array}$ \\
\hline
\end{tabular}




\begin{tabular}{|l|l|}
\hline Otras & $\begin{array}{l}\text { Taller de expresión plástica manual, Fundamentos de la Educación, Historia de la Edu- } \\
\text { cación, Desarrollo profesional docente, Currículo Educación parvularia. Diseño Curricu- } \\
\text { lar, Didáctica matemáticas y ciencias, Teoría Evaluativa, Expresión Corporal, Exploración } \\
\text { e Indagación en la Infancia, Psicomotricidad, Expresión musical, Expresión Teatral, Aná- } \\
\text { lisis de procesos evaluativos: Primer Ciclo Educación Parvularia, Lenguajes artísticos, } \\
\text { Literatura infantil, Didáctica de las Matemáticas, Práctica: El Educador de Párvulos, Edu- } \\
\text { cación y Vocación, Enseñanza y aprendizaje, Pedagogía de las Ciencias Naturales, Di- } \\
\text { dáctica Iniciación Matemática. }\end{array}$
\end{tabular}

Fuente: Elaboración Propia. Nota: *Algunos profesores imparten más de una asignatura

\section{Resultados}

\section{Percepción de los profesores sobre los medios y su enseñanza}

Ideas sobre los medios

La mayoría de los profesores tienen una visión crítica de cómo informan los medios de comunicación, ya que 27 de ellos está en desacuerdo o muy en desacuerdo con la idea de que los medios informan de manera objetiva los hechos. Los profesores TIC rechazan totalmente esta idea, mientras el rechazo no es unánime en los profesores de Lenguaje y Comunicación. Respecto de la idea de que los medios pueden ayudar a la autonomía personal y la transformación social, la mayoría de los profesores muestra una visión positiva de las oportunidades que ofrecen los medios puesto que 25 de ellos están de acuerdo o muy de acuerdo con esta idea. Los profesores de asignaturas TIC y los de asignaturas de Lenguaje y Comunicación la suscriben de forma unánime.

La idea de que los medios son el principal vehículo de socialización en la actualidad es apoyada por una mayoría. No obstante, en comparación de las tres ideas expuestas es la que genera menos consenso ya que solo 20 de los profesores están de acuerdo o muy de acuerdo.. Finalmente, respecto de la idea de que la población debiera recibir una formación en medios de comunicación, la mayoría de los profesores está de acuerdo o muy de acuerdo con esta idea, generando un total acuerdo en los profesores TIC y los de Lenguaje y Comunicación.

Tabla 3: Grado de acuerdo de profesores universitarios con ideas sobre los medios de comunicación en la sociedad

\begin{tabular}{|l|l|l|l|l|l|l|}
\hline $\begin{array}{l}\text { Ideas sobre los } \\
\text { medios } \\
\text { comunicación }\end{array}$ & $\begin{array}{l}\text { Muy } \\
\text { desacuerdo }\end{array}$ & $\begin{array}{l}\text { En } \\
\text { desacuerdo }\end{array}$ & $\begin{array}{l}\text { De } \\
\text { acuerdo }\end{array}$ & $\begin{array}{l}\text { Muy de } \\
\text { acuerdo }\end{array}$ & $\begin{array}{l}\text { No lo } \\
\text { sé }\end{array}$ & Total \\
\hline $\begin{array}{l}\text { Son el principal vehículo } \\
\text { de socialización en la } \\
\text { actualidad }\end{array}$ & 2 & 8 & 13 & 7 & 0 & 30 \\
\hline
\end{tabular}




\begin{tabular}{|c|c|c|c|c|c|c|}
\hline $\begin{array}{l}\text { Pueden ayudar a la } \\
\text { autonomía personal y la } \\
\text { transformación } \\
\text { social }\end{array}$ & $0 \%$ & $\begin{array}{l}4 \\
13,33 \%\end{array}$ & $\begin{array}{l}18 \\
60 \%\end{array}$ & $\begin{array}{l}7 \\
23,33 \%\end{array}$ & $\begin{array}{l}1 \\
3,33 \%\end{array}$ & 30 \\
\hline $\begin{array}{l}\text { Informan de manera } \\
\text { objetiva sobre los hechos } \\
\text { que suceden }\end{array}$ & $16,67 \%$ & $\begin{array}{l}22 \\
73,33 \%\end{array}$ & $6,67 \%$ & $0 \%$ & $\begin{array}{l}1 \\
3,33 \%\end{array}$ & 30 \\
\hline
\end{tabular}

\section{Influencia de los medios}

La mayoría de los profesores está en desacuerdo o muy en desacuerdo con la idea de que los medios ejercen menos influencia cuando su función es solo entretener o ayudar a "pasar el rato", con 24 menciones. Los profesores de Lenguaje y Comunicación son los que más rechazan esta idea. Respecto de la entretención, la mayoría está de acuerdo o muy de acuerdo con la idea de que los medios ejercen una influencia mayor cuando apelan solo a lo emocional e inconsciente de las personas, con 21 menciones. Los profesores de Lenguaje y Comunicación son los que muestran un total acuerdo (muy de acuerdo o de acuerdo) con esta idea. En cuanto a lo racional, la mayoría está de acuerdo o muy de acuerdo con la idea de que los medios ejercen una influencia mayor cuando entregan elementos cognitivos, con 17 menciones, siendo de las tres ideas propuestas la que genera menos consenso. Los profesores TIC son los que más apoyan esta idea, mientras los de Lenguaje y Comunicación los que menos la apoyan.

Tabla 4: Grado de acuerdo de los profesores universitarios con ideas sobre la influencia de los medios de comunicación en las personas

\begin{tabular}{|c|c|c|c|c|c|c|}
\hline $\begin{array}{l}\text { Ideas sobre influencia de } \\
\text { los medios }\end{array}$ & $\begin{array}{l}\text { Muy en } \\
\text { desacuerdo }\end{array}$ & $\begin{array}{l}\text { En } \\
\text { desacuerdo }\end{array}$ & $\begin{array}{l}\text { De } \\
\text { acuerdo }\end{array}$ & $\begin{array}{l}\text { Muy de } \\
\text { acuerdo }\end{array}$ & $\begin{array}{l}\text { No lo } \\
\text { sé }\end{array}$ & Total \\
\hline $\begin{array}{l}\text { Ejercen menos influencia } \\
\text { cuando su función es solo } \\
\text { entretener o ayudar a "pasar el } \\
\text { rato" }\end{array}$ & $13,33 \%$ & $\begin{array}{l}17 \\
56,67 \%\end{array}$ & $23,33 \%$ & $6,67 \%$ & $0 \%$ & 30 \\
\hline $\begin{array}{l}\text { Ejercen una influencia mayor } \\
\text { cuando apelan solo a lo } \\
\text { emocional e inconsciente de } \\
\text { las personas }\end{array}$ & $3,33 \%$ & $16,67 \%$ & $60 \%$ & $20 \%$ & $0 \%$ & 30 \\
\hline $\begin{array}{l}\text { Ejercen una influencia mayor } \\
\text { cuando entregan elementos } \\
\text { cognitivos y racionales }\end{array}$ & $0 \%$ & $\begin{array}{l}12 \\
40 \%\end{array}$ & $\begin{array}{l}11 \\
36,67 \%\end{array}$ & $\begin{array}{l}6 \\
20 \%\end{array}$ & $\begin{array}{l}1 \\
3,33 \%\end{array}$ & 30 \\
\hline
\end{tabular}




\begin{tabular}{|l|l|l|l|l|l|l|}
\hline $\begin{array}{l}\text { Las personas deberían recibir } \\
\text { una formación que les permita }\end{array}$ & 1 & 1 & 8 & 19 & 1 & 30 \\
$\begin{array}{l}\text { relacionarse de forma positiva } \\
\text { con los medios. }\end{array}$ & $3,33 \%$ & $3,33 \%$ & $26,67 \%$ & $63,33 \%$ & $3,33 \%$ \\
\hline
\end{tabular}

\section{Incorporación de la Educación Mediática en los planes de estudio}

La idea de que la enseñanza de los medios de comunicación debe estar incorporada en los planes de estudios de las carreras de Educación Parvularia genera unanimidad entre los profesores. Sin embargo, existen distintas visiones acerca del cómo debiera implementarse. Un poco más de la mitad de los profesores señala que la educación mediática debiera estar presente como un contenido transversal. La idea de asignatura especifica es apoyada por siete profesores, mientras seis señalan que solo tiene que ser un contenido dentro de una asignatura. Al respecto, existen opiniones divididas entre los profesores de las asignaturas TIC, que piensan que debiera ser una asignatura específica y un contenido transversal y los de Lenguaje y Comunicación que señalan, principalmente, que debiera ser un contenido transversal.

Tabla 5: Percepción sobre la forma de incorporar la enseñanza de los medios de comunicación en los planes de estudios de las carreras de educación parvularia

\begin{tabular}{|l|l|l|}
\hline $\begin{array}{l}\text { Forma de incorporar la enseñanza de } \\
\text { los medios en la universidad }\end{array}$ & Número & Porcentaje \\
\hline Una asignatura específica & 3 & $10 \%$ \\
\hline Un contenido de una asignatura & 6 & $20 \%$ \\
\hline Un contenido transversal & 16 & $53,33 \%$ \\
\hline No debiera ser incorporada & 0 & $0 \%$ \\
\hline Otro (especifique) & 5 & $16,67 \%$ \\
\hline Total & 30 & $100 \%$ \\
\hline
\end{tabular}

Enseñanza de los medios en el jardín infantil

Existe una apertura a que los medios de comunicación estén presentes en los jardines infantiles. La mayoría de los profesores (23 de 30) piensa que sí sería relevante enseñar sobre medios de comunicación a los niñas y niños en los jardines infantiles. La idea que genera mayor consenso es que se le debiera enseñar un uso adecuado de los medios. Este uso adecuado incluye mediar el uso que ya hacen los niños fuera del Jardín, utilizar los medios para expresiones artísticas y para sociabilizar, buscar información y resolver problemas, trabajar con los formatos más usados como los smartphones y enseñarles un uso adecuado del tiempo de exposición.

"Las plataformas y soportes de la interfaz cambian permanentemente, por lo que creo se debería trabajar temporalmente en la relación con el formato que se está usando en cada momento (hoy smarthphones)" (Profesor asignatura TIC). 
En segundo lugar, aparecen las ideas de enseñar a los niños un análisis crítico de las pantallas y a no ser vulnerables frente a los medios. La primera incluye que sepan distinguir y ser críticos con los contenidos y distinguir quién crea el mensaje, mientras la segunda idea apunta al autocuidado respecto del tiempo de exposición y los abusos de los medios. En menor medida encontramos las ideas de enseñar a los niños a buscar información, distinguir e identificar los medios y que sean un recurso para aprender.

"Pienso que se les debe enseñar a ser críticos con los contenidos a no ser vulnerables a la construcción social que tratan de crear los medios según sus propias ideologías" (Profesor Lenguaje y Comunicación). "Me parece importante enseñar a niños, niñas, padres y apoderados, sobre los usos y abusos de la televisión, por ejemplo. La información que se entrega. Lo que deben o no ver los niños, lo que se debe conversar en familia" (Profesor de otra asignatura).

Por otro lado, hay siete profesores que dijeron que no es relevante enseñar a los niños sobre medios de comunicación y que éstos deben mantenerse alejados del jardín infantil. Debido a esto, solo se debe mediar su uso en los niños y enseñar a los padres sobre lo que deben ver los niños. Además, se señaló la importancia de que las educadoras conozcan los efectos en el desarrollo de los niños.

"Creo que la aproximación a medios de comunicación en el Jardín Infantil debe ser secundaria. Las educadoras y educadores deben conocer el efecto que tienen las pantallas en el desarrollo cerebral de un niño a temprana edad (Profesor de Lenguaje y Comunicación)".

"No me parece relevante (enseñar a los niños). Me parece relevante que se enseñe a los padres sobre los efectos que éstos pueden tener en sus hijos y la incidencia que tienen en distintas áreas; cognitiva, desarrollo imaginación, emocional" (Profesor de otra asignatura).

Los resultados muestran que la totalidad de los profesores de asignaturas TIC piensan que sí se debiera enseñar a los niños y niñas sobre medios de comunicación en los jardines infantiles, apuntando principalmente a aprovechar el potencial de los medios, identificarlos y a tener una mirada crítica de ellos. "Se les debe enseñar a utilizarlos para crear expresiones artísticas (imagen, audio, forma, etc.) y para realizar actividades cooperativas que propendan a crear conciencia de metas comunes y del valor tanto del aporte individual (para fortalecer la auto-confianza) como del de los demás (...) Finalmente, creo que, si es posible, los educadores deben llevar a los niños a vislumbrar el potencial creativo y práctico sin límite de los medios de comunicación" (Profesor asignatura TIC).

Por el contrario, la mayoría de los profesores de Lenguaje y Educación señalan que no se debiera enseñar sobre medios de comunicación a los niños y niñas en el jardín infantil. En todos existe una mirada negativa de los medios, puesto que en el único caso que se señala que sí se les debe enseñar se refiere a no ser vulnerables con las ideologías que contienen los mensajes de los medios. Los que afirman que no se debiera enseñar buscan preservar el jardín infantil como un lugar alejado de la "contaminación" que producen los medios.

"No estoy de acuerdo con que en el jardín se utilicen medios de comunicación tales como televisión, internet, vídeos, porque ya los padres, muchos, no todos, abusan de estos medios en el hogar. Creo que quienes necesitan educación sobre esto son los padres, que programas podrían ver los niños, cuánto tiempo, por qué restringir su uso en niños pequeños, como su uso debe estar supervisado por adultos. Estamos invadidos de medios de comunicación, y el jardín debería ser un ambiente "limpio" de esto y ofrecer experiencias de aprendizaje que estén fuera del alcance de los padres (Profesor de Lenguaje y Comunicación). 


\section{Perfiles de profesores}

A partir de los resultados obtenidos hemos configurado perfiles de los profesores según las asignaturas que enseñan y su percepción de los medios. Existe coincidencia en las ideas sobre los medios, en especial en que pueden ayudar a la autonomía personal, que no informan objetivamente y que ejercen una influencia mayor cuando apelan solo a lo emocional e inconsciente de las personas. También los profesores concuerdan en que las personas deberían recibir una formación que les permita relacionarse de forma positiva con los medios.

Las mayores diferencias entre ellos se dan en la forma en que se debe incorporar la enseñanza de los medios en los planes de estudio de las futuras educadoras y respecto de si se debe incorporar la educación mediática en los jardines infantiles, es decir, en la implementación de esta educación.

\section{Profesores TIC}

Este grupo apoya unánimemente la necesidad de que la población reciba una educación mediática. Respecto a las ideas sobre los medios, los profesores TIC son los que tienen mayor claridad de que los medios no informan objetivamente y que pueden ayudar a la autonomía personal. Reconocen mayoritariamente la influencia de los medios cuando apelan a lo emocional y al inconsciente de las personas.

Valoran, pero con menos fuerza, la influencia cuando las personas recurren a los medios para "pasar el rato" y cuando apelan fundamentalmente a elementos racionales, y son el segundo grupo en apoyar la idea de que son el principal vehículo de socialización.

Los profesores TIC son los que más apoyan la idea de que la enseñanza de los medios debiera ser una asignatura especifica en los planes de estudio de la formación docente de la educación infantil. Además, todos suscriben la idea de que se debiera enseñar a los niños y niñas sobre medios de comunicación en los jardines infantiles, especialmente al uso de los recursos tecnológicos, a tener una mirada crítica y a identificar los medios.

\section{Profesores de Lenguaje y Comunicación}

Todos los profesores de este grupo expresan la necesidad de que la población reciba una educación mediática. Respecto a las ideas sobre los medios, los profesores que imparten asignaturas de Lenguaje y Comunicación son los que tienen mayor claridad de la importancia e influencia que ejercen los medios a través de aspectos emocionales y de la entretención. Al igual que los profesores TIC, todos están de acuerdo con la idea de que los medios pueden ayudar a la autonomía personal y la transformación social.

Son los que muestran un menor rechazo a la idea de que los medios informan de manera objetiva y son los que menos apoyan la idea de que los medios ejercen una influencia mayor cuando entregan elementos cognitivos, lo que es consistente con su valoración de lo emocional. A diferencia de los otros grupos, no apoyan la idea de que los medios son el principal vehículo de socialización en la actualidad. 
Los profesores de Lenguaje y Comunicación están relativamente a favor de la idea de que los medios de comunicación deben estar incorporados en los planes de estudios como contenido transversal de la formación docente de la educación infantil. Uno de los puntos más distintivos de este grupo es que la mayoría señala que no se debiera enseñar sobre medios de comunicación a los niños y niñas en el jardín infantil. En todos existe una mirada negativa de los medios, puesto que en el único caso que se señala que sí se les debe enseñar se refiere a no ser vulnerables con las ideologías que contienen los mensajes de los medios. Los que señalan que no se debiera enseñar buscan preservar el jardín infantil como un lugar "limpio de los medios".

\section{Discusión y Conclusiones}

Los resultados de la investigación nos permiten establecer distintos niveles de análisis. En un nivel general podemos apreciar que los profesores universitarios que forman educadores (as) poseen un conocimiento adecuado del rol de los medios en la sociedad, reconociendo la importancia de los aspectos emocionales, de la entretención y la apelación al inconsciente que realizan los medios.

Sin embargo, en lo específico, carecen de la profundidad necesaria para superar el reduccionismo de la educación mediática a la alfabetización digital que existe en el sistema educacional, lo cual se expresa en enseñar solamente un uso adecuado de los medios. Ninguno de los que estaba de acuerdo con enseñar sobre medios de comunicación en los jardines infantiles mencionó la importancia de que los niños reconozcan las emociones que le producen la interacción con las pantallas o que puedan reflexionar ante los productos audiovisuales que los entretienen.

Es positivo que las percepciones sobre los medios de comunicación de los profesores universitarios sean afines a las ideas generales que se han reflexionado desde la educación mediática durante las últimas décadas. Saben que los medios no son objetivos cuando informan y reconocen su potencia para ayudar a la autonomía personal y la transformación social. Incluso, a diferencia de los expertos en educación mediática (Ferrés, Masanet y Marta, 2013), los profesores tendrían una mayor valoración por los aspectos emocionales, el entretenimiento y los procesos mentales inconscientes que ocurren frente a las pantallas. Las ideas sobre los medios suscritas por los profesores quedan en entredicho cuando se les pregunta por implementar la educación mediática en la formación docente y en los jardines infantiles. Casi la totalidad de los profesores señala que la población debiera recibir una formación en educación mediática, pero ellos no se sentirían responsables de implementarla.

En su mayoría los profesores piensan que los medios debieran ser un contenido transversal de los planes de estudio, lo que nos lleva a pensar que no existe un interés por incorporar el estudio de los medios, ya que habitualmente los contenidos transversales terminan diluyéndose en los cursos. Lo anterior se corresponde con la formación en TIC que han recibido más de la mitad de los profesores, donde se promueve el uso de la tecnología como un recurso que sirve para motivar a los estudiantes y no como un objeto de estudio.

Las tradiciones epistemológicas de la relación entre educación y medios de comunicación están presentes en estas percepciones, permitiéndonos realizar aproximaciones a perfiles de profesores y proponer acciones desde la educación mediática. Las percepciones sobre la enseñanza de los medios en los jardines infantiles 
evidenciaron la presencia de las tradiciones apocalíptica y apologética en los profesores universitarios de la educación infantil chilena que han participado de este estudio.

Los apocalípticos los encontramos preferentemente en los profesores de Lenguaje y Comunicación que muestran una visión negativa de la presencia de los medios de comunicación en el jardín infantil. El paradigma inoculativo se mantiene vigente cuando aparecen las ideas de mantener el centro educativo como un lugar "limpio" de los medios y de la responsabilidad de los padres y las educadoras desde la responsabilidad de conocer los efectos dañinos de los medios y controlar los tiempos de exposición de los niños, repitiendo así, los argumentos expresados por los reivindicadores (Buckingham, 2013) que alertan sobre los peligros de los medios.

Dentro de los que piensan que sí se debe enseñar sobre los medios, también encontramos esta visión de proteger a los niños de los medios. Se encuentra en la idea de que los niños aprendan a cuidarse de los abusos de los medios, incorporando el diálogo con la familia. En la visión crítica de los medios, que también hemos incluido en la tradición apocalíptica, los profesores postulan que se debe enseñar a no creer lo que se muestra como una verdad, siendo críticos con los contenidos de los mensajes mediáticos.

Los apologistas se encuentran en los profesores TIC. Los distingue una visión de que los medios pueden ser positivos en la formación de los niños. En los profesores TIC se expresa la promoción de la competencia digital en la importancia de enseñar sobre los medios de comunicación para buscar información y distinguir la valides de cada medio en tanto fuente legitima.

Sin embargo, la idea que más se reitera es la del uso de los medios como recurso. De esta forma se reproduce algunas de las ideas de hace una década respecto del uso de las TIC, lo que ha llevado a un reduccionismo de la educación mediática a la dimensión del uso y la tecnología. El problema de esta visión es que olvida el viejo, pero aún vigente, aforismo de Mc Luhan de que el medio es el mensaje o, dicho de otra forma, se omite que para usar correctamente el medio es necesario entenderlo en toda su complejidad. Este reduccionismo muestra signos de agotamiento y ha provocado revisiones, como por ejemplo el de la política digital en educación escolar en Chile. Tampoco se toma en cuenta que los factores emocionales, estéticos y de interacción son fundamentales para aprender sobre los medios de comunicación y sobre la tecnología.

Los resultados nos muestran que el tipo de asignatura que enseñan los profesores influye en la percepción que tienen sobre los medios de comunicación, ya que respondería a alguna de las tradiciones epistemológicas de la relación entre educación y medios de comunicación. Los profesores que enseñan sobre Lenguaje y Comunicación responden más a la tradición apocalíptica de los medios, en especial al paradigma inoculativo, mientras los de TIC siguen la tradición apologista en su versión de la competencia digital. Dentro de las limitaciones de este estudio está que se trabajó con una muestra pequeña que solo permite realizar una aproximación, por lo que se debe seguir investigando estos resultados.

Proponemos que las próximas investigaciones profundicen en las actitudes, conocimientos y propuestas sobre educación mediática de los profesores universitarios que forman educadores (as) que imparten las asignaturas TIC y las de Lenguaje y Comunicación, ya que son los agentes claves en las actitudes y conocimientos sobre los medios de comunicación que los futuros profesores llevarán al aula. 


\section{Bibliografía}

Aguaded-Gómez, M.C., López, L. \& Cruz-Díaz, R. (2015). Fortalezas y debilidades de la competencia mediática en la enseñanza universitaria: guías docentes y perfil del profesorado. En Ferrés y Masanet (Eds.). La educación mediática en la universidad española. Barcelona: Gedisa.

Buckingham, D. (2013). La Infancia Materialista. Crecer en la cultura consumista. Madrid: Morata.

Consejo Nacional de Televisión (CNTV) de Chile (2017). IX Encuesta Nacional de Televisión de Chile.

De Fontcuberta, M. (2009). Propuestas para la formación en educación en medios en profesores chilenos. Comunicar, 32. 201-207. doi:10.3916/c32-2009-03-001

Dortman, A. \& Mattelard, A. (2005). Para leer al pato Donald. México: Siglo XXI.

Fedorov, A. (2011). Alfabetización mediática en el mundo. Infoamérica. Revista Iberoamericana de Comunicación, 5. 7-23. Recuperado de https://www.infoamerica.org/icr/n05/fedorov.pdf

Ferrés, J. \& Piscitelli, A. (2012). La competencia mediática: propuesta articulada de dimensiones e indicadores. Comunicar, 38. 75-82. doi:10.3916/C38-2012-02-08

Ferrés, J., Masanet, M. J. \& Marta, C. (2013). Neurociencia y educación mediática: carencias en el caso español. Historia y Comunicación Social, 18. 129-144. doi:10.5209/rev_HICS.2013.v18.44317

Ferrés, J, Masanet, M. J. \& Blanco, S. (2014). La educación mediática como carencia. En Ilana Eleá (Ed.) Agentes e vozes. Um panorama da mídia-educação no Brasil, Portugal e Espanha. 265-272. Nordicom: University of Gothenburg.

Freire, P. (2005) Pedagogía del oprimido. México: Siglo XXI.

Fuenzalida, V. (2005). Expectativas Educativas de las audiencias televisivas. Bogotá: Norma.

Fuenzalida, V. (2016). La Nueva Televisión Infantil. Santiago de Chile: Fondo de Cultura Económica.

Gutiérrez, A. \& Tyner, K. (2012). Educación para los medios, alfabetización mediática y competencia digital. Comunicar, 38. 31-39. doi: 10.3916/C38-2012-02-03

Jenkins, H. (2008). Convergence culture: La convergencia de la cultura de los medios de comunicación. Barcelona: Paidós.

Kaplún, M. (2002). Una pedagogía de la comunicación (el comunicador popular). La Habana: Editorial caminos.

López, L., \& Aguaded, M. C. (2015). La docencia sobre alfabetización mediática en las facultades de Educación y Comunicación. Comunicar, 44, 187-195. doi: 10.3916/C44-2015-20

Mineduc (2012). Estándares Orientadores para Carreras de Educación Parvularia. Santiago: Ministerio de Educación de Chile. Recuperado de: http://ww2.educarchile.cl/UserFiles/P0001/Image/Estudiantes pedagogia/estud pedagogia/estan dares Fid/estandar parvularia.pdf

Mineduc (2014). Informe de Resultados SIMCE TIC 20 Medio 2013. Santiago: Ministerio de Educación de Chile. Recuperado de: http://www.enlaces.cl/wp-content/uploads/informe-resultados-final-16-122014.pdf

Mineduc (2016). Bases Curriculares 70 básico a $2^{\circ}$ medio. Santiago: Ministerio de Educación de Chile. Recuperado de: http://www.curriculumnacional.cl/inicio/ 
Mineduc (2017). Recomendaciones para una Política Digital en Educación Escolar. Santiago: Ministerio de Educación de Chile. Recuperado de: http://cpce.udp.cl/wpcontent/uploads/2017/09/Recomendaciones-Poli\%CC\%81tica-Digital.pdf

Palmer, S. (17 de noviembre de 2016). SCREEN LOCK As screen addiction is becoming a growing problem for our kids, here's how to stop them from having an iPaddy. The Sun. Recuperado de: https://www.thesun.co.uk/living/2202225/how-to-prevent-the-ipaddy-screen-addiction-inchildren/

Pereira, S.; Pinto, M.; Madureira, E. J.; Pombo, T. \& Guedes, M. (2014). Referencial de Educação para os Media para a Educação Pré-escolar, o Ensino Básico e o Ensino Secundário. Ministério da Educação e Ciência de Portugal.

Purcell, F. (2011). Cine y censura en Chile: Entre lo local y lo transnacional, 1910-1945. Atenea(Concepción), 503, 187-201. doi: 10.4067/S0718-04622011000100010

Resolución 2008/2129 (INI) del Parlamento Europeo (24 de noviembre de 2008). Resolución sobre la alfabetización de los medios de comunicación en un mundo digital. Recuperado de: http://www.europarl.europa.eu/sides/getDoc.do?pubRef=-//EP//TEXT+REPORT+A6-2008$\underline{0461+0+\mathrm{DOC}+\mathrm{XML}+\mathrm{V} 0 / / \mathrm{ES}}$

Unesco (1982). Declaración de Grünwald sobre la Educación relativa a los Medios de Comunicación. 22 de enero de 1982. Recuperado de: http://www.unesco.org/education/pdf/MEDIA_S.PDF

Unesco (2007). Paris Agenda or 12 Recommendations for Media Education. Recuperado de: http://www.diplomatie.gouv.fr/IMG/pdf/Parisagendafin_en.pdf

Valencia-Molina, T., Serna-Collazos, A., Ochoa-Angrino, S., Caicedo-Tamayo, A. M., Montes-González, J. A., \& Chávez-Vescance, J. D. (2016). Competencias y estándares TIC desde la dimensión pedagógica: una perspectiva desde los niveles de apropiación de las TIC en la práctica educativa docente. Cali: Ponitificia Universidad Javeriana.

Wilson, C., Grizzle, A., Tuazon, R., Akyempong, K., \& Cheung, C.-K. (2011). Alfabetización Mediática e Informacional. Curriculum para profesores. Recuperado de: http://unesdoc.unesco.org/images/0021/002160/216099S.pdf 